Bio - grafia. Escritos sobre la Biología y su Enseñanza. ISSN 2027

Edición Extraordinaria. p.p. 366 - 376

Memorias del VIII Encuentro Nacional de Experiencias en Enseñanza de la Biología y la Educación Ambiental. III Congreso Nacional de Investigación en Enseñanza de la Biología.

\title{
PRÁCTICAS DE LABORATORIO EN EDUCACIÓN AMBIENTAL: UNA EXPERIENCIA LÚDICO CIENTÍFICA EN EL AULA
}

\section{LABORATORY PRACTICE IN ENVIRONMENTAL EDUCATION: A SCIENTIFIC LEISURE EXPERIENCE IN THE CLASSROOM}

\author{
Adriana $C$. Rojas $C{ }^{1}$ \\ Oscar D. Pérez M. ${ }^{2}$
}

Resumen: Esta investigación cualitativa se basó en la enseñanza de la Educación Ambiental (EA) a través de la experimentación principalmente con el tema de la lluvia ácida, la cual se basó en las prácticas de laboratorio, posteriormente se aplicó una cartografía social (CS) para reconocer la problemática en los contextos locales de los estudiantes de básica secundaria y finalmente se concluyó con la elaboración de Resúmenes Analíticos Educativos (RAE) para identificar la argumentación científica y ambiental. En este sentido, se contribuyó al desarrollo de consciencia ecológica y el pensamiento crítico entorno a las diferentes problemáticas ambientales que viven los estudiantes, en especial la relación con la lluvia ácida, además las prácticas lograron generar discusiones reflexivas acerca las prácticas humanas en la afectación del territorio, además permitió reflexionar de las acciones colectivas para su conservación y recuperación.

ABSTRACT: This qualitative research was based on the teaching of environmental education (EA) through experimentation mainly with the issue of acid rain, which was based on the labs, then he applied a social mapping (CS) to recognize the

\footnotetext{
${ }^{1}$ Licenciada En Química, Universidad Distrital Francisco José De Caldas. Magister En Educación (En Formación) Universidad Pedagógica Nacional, Teléfono 313-3242205. Correos caro.rc1723@gmail.comarojasc@redp.edu.co Bogotá, Colombia. Se presenta el escrito bajo la Modalidad: Artículo de investigación científica y tecnológica.

2 Licenciado en Química de la Universidad Pedagógica Nacional, Tecnólogo en Saneamiento Ambiental de la Universidad Distrital Francisco José De Caldas, Gestor en Desarrollo Sostenible y Ambiental de la ESAP. Teléfono 3142834629. Correos oscardanielperez26@gmail.com, dqu878 operez@pedagogica.edu.co. Bogotá, Colombia. Se presenta el escrito bajo la Modalidad: Artículo de investigación científica y tecnológica.
} 
Bio - grafia. Escritos sobre la Biología y su Enseñanza. ISSN 2027

Edición Extraordinaria. p.p. 366 - 376

Memorias del VIII Encuentro Nacional de Experiencias en Enseñanza de la Biología y la Educación Ambiental. III Congreso Nacional de Investigación en Enseñanza de la Biología.

problem in local contexts of basic and high school students finally concluded with the development Educational Abstracting (RAE) to identify scientific and environmental arguments. In this sense, he contributed to the development of ecological awareness and critical thinking around the different environmental problems experienced by students, especially regarding acid rain, plus the practices able to generate thoughtful discussions about human practices in the involvement territory, also allowed reflection of collective action for their conservation and recovery.

PALABRAS CLAVES: Educación Ambiental, Ciencia, Experimentación, Lluvia Ácida KEYWORDS: Environment Education, Sciences, Experimentation, Acid Rain

\section{INTRODUCCIÓN}

La propuesta de investigación fue planteada para identificar y problematizar a los estudiantes de secundaria en torno a las problemáticas ambientales actuales en este caso la lluvia ácida, por lo tanto fue importante la identificación de las causas, las consecuencias y las posibles soluciones, la investigación se basó en experimentos sencillos en el aula que involucran de manera significativa y pedagógica los principios de la Educación Ambiental (EA), la Química y la Biología. Estas prácticas vivenciales de laboratorio fortalecieron el aprendizaje activo además de generar conciencia en los estudiantes de las problemáticas ambientales a través de actividades lúdico científicas.

Asimismo, la enseñanza y el aprendizaje desde la EA y las ciencias naturales, favorece el desarrollo de habilidades cognitivas en la búsqueda de que los estudiantes puedan transformar su contexto así como mejorar la calidad de vida. Por tal motivo, el fortalecimiento del pensamiento crítico se buscó en los Resúmenes Analíticos Educativos (RAE) desde la EA y la educación científica con el fin de preparar futuros ciudadanos con conocimientos y capacidades que les permitan tomar decisiones en pro de su contexto entendiendo los avances de la Ciencia y la Tecnología. 
Bio - grafia. Escritos sobre la Biología y su Enseñanza. ISSN 2027

Edición Extraordinaria. p.p. 366 - 376

Memorias del VIII Encuentro Nacional de Experiencias en Enseñanza de la Biología y la Educación Ambiental. III Congreso Nacional de Investigación en Enseñanza de la Biología.

Uno de los objetivos fundamentales de esta investigación es propiciar escenarios de reflexión y participación activa, donde los estudiantes puedan construir colectivamente el saber de la Educación Ambiental en particular la lluvia ácida como temática propuesta. En concordancia, durante ese ejercicio fue fundamental indagar la finalidad como educadores para motivar a los estudiantes a aprender de manera lúdica y a la vez científica, sin embargo es necesario reconocer el hecho que existen limitaciones como por ejemplo que no podemos reproducir de manera exacta las problemáticas o fenómenos ambientales en el aula, entonces se buscó presentar a los estudiantes simulaciones experimentales que nos permitan acercarnos a dichas problemáticas, tomando también como herramientas el uso de la tecnología, debates sobre con los RAE, actividades de cartografía social junto a las prácticas de laboratorio.

En cuanto a la revisión de los antecedentes para la formulación de la investigación se tuvo presente el documento «La Dimensión Ambiental: un reto para la educación de la nueva sociedad Proyectos escolares», escrito por Maritza Torres (1997) en donde se rescatan consideraciones como que el proceso debe permitir a los individuo comprender las relaciones de interdependencia con su entorno, a partir del conocimiento reflexivo y crítico de su realidad biofísica, social, política económica y cultural. Asimismo, es importante para la investigación tomar los aportes conceptuales del documento en cuanto a que debe ser un campo de investigación constante y fundamental, preocuparse por la construcción de un proyecto de sociedad en el cual la calidad de vida sea eje fundamental y hacer parte del proyecto de transformación del sistema educativo, la reformulación del quehacer pedagógico y didáctico y la elaboración de modelos para la construcción del conocimiento. En concordancia, surge la importancia de fortalecer la visión integradora como resultado de las interacciones dinámicas de los sistemas sociales y naturales con todos los saberes para comprender la problemática ambiental como global y sistemática, es que no se debe dejar solo la tarea de la EA a los proyectos PRAE, sino que se debe fortalecer desde cada una de las áreas disciplinares por lo tanto se plantea la experiencia lúdico científica en el aula y así una apropiación de una problemática global como lo es la lluvia ácida. 
Bio - grafia. Escritos sobre la Biología y su Enseñanza. ISSN 2027

Edición Extraordinaria. p.p. 366 - 376

Memorias del VIII Encuentro Nacional de Experiencias en Enseñanza de la Biología y la Educación Ambiental. III Congreso Nacional de Investigación en Enseñanza de la Biología.

\section{METODOLOGÍA}

El trabajo de investigación, se basó en el enfoque cualitativo, por medio de instrumentos de investigación interpretativa, considerando su pertinencia para comprender los procesos de metacognición y práctica educativa (Carr y Kemmis, 1988). Además, la implementación de este método cualitativo de investigación estuvo retroalimentado por la observación participante, lo que para el análisis resulta una participación como investigador de manera abierta en la vida diaria de las personas durante un período de tiempo, se logró observar qué sucedía y las actividades que realizan.

\begin{tabular}{|c|c|}
\hline FASES & TÉCNICAS \\
\hline 1. Revisión de Antecedentes & - Documental \\
\hline $\begin{array}{l}\text { 2. Experimentación lluvia } \\
\text { ácida }\end{array}$ & $\begin{array}{l}\text { - Practica de laboratorio } \\
\text { - Observación Particular }\end{array}$ \\
\hline $\begin{array}{l}\text { 3. Actividades reconocimiento } \\
\text { territorial }\end{array}$ & $\begin{array}{c}\text { - Resúmenes Analíticos Educativos } \\
\text { - Cartografía Social }\end{array}$ \\
\hline $\begin{array}{l}\text { 4. Procesamiento de la } \\
\text { información }\end{array}$ & $\begin{array}{lc}\text { - } & \text { Documental } \\
\text { - } & \text { Matrices cualitativas } \\
\text { - } & \text { Categorización }\end{array}$ \\
\hline
\end{tabular}

\section{RESULTADOS Y DISCUSIÓN}

\section{REVISIÓN DE ANTECEDENTES}

Durante esta fase de la investigación se buscó caracterizar las fortalezas y debilidades de la proyección en este tema, teniendo como fundamento cada una de las dimensiones, parámetros y perfiles que se deben tener en cuenta para la 
Bio - grafia. Escritos sobre la Biología y su Enseñanza. ISSN 2027

Edición Extraordinaria. p.p. 366 - 376

Memorias del VIII Encuentro Nacional de Experiencias en Enseñanza de la Biología y la Educación Ambiental. III Congreso Nacional de Investigación en Enseñanza de la Biología.

formulación de acciones en la educación ambiental. Para lo cual se utilizó una miscelánea de herramientas de investigación interpretativa y una serie de informaciones de fuentes primarias y secundarias en las cuales se encontraban los planteamientos de González (2003), con los documentos juegos de educación ambiental laboratorio de productos químicos.

\section{EXPERIMENTACIÓN "LLUVIA ÁCIDA"}

En esta fase de la investigación se llevó a cabo las prácticas de laboratorio con el fin de demostrar los dañinos efectos de la lluvia ácida, además de aprovechar para resaltar contenidos temáticos de química como Ácidos, Oxiácidos, contaminación ambiental, pH y tipos de reacción. En el tema de lluvia ácida y calentamiento global uno de los temas socio científicos más importante para tratar en la educación ambiental, se llevó a cabo a través de la argumentación científica contextualizada estableciendo la conceptualización de la lluvia ácida.

La experimentación se basó en diferentes actividades como el Video - lluvia ácida con el objetivo de aproximar a los estudiantes a la problemática sus causas y efectos, posteriormente se llevó a cabo la lectura: Lluvia Ácida La Amenaza Invisible, departamento de conservación ambiental, George E. Pataki, (2002). con el objetivo de Debatir y reflexionar acerca de las posibles soluciones de disminución de la lluvia ácida. 
Bio - grafia. Escritos sobre la Biología y su Enseñanza. ISSN 2027

Edición Extraordinaria. p.p. 366 - 376

Memorias del VIII Encuentro Nacional de Experiencias en Enseñanza de la Biología y la Educación Ambiental. III Congreso Nacional de Investigación en Enseñanza de la Biología.

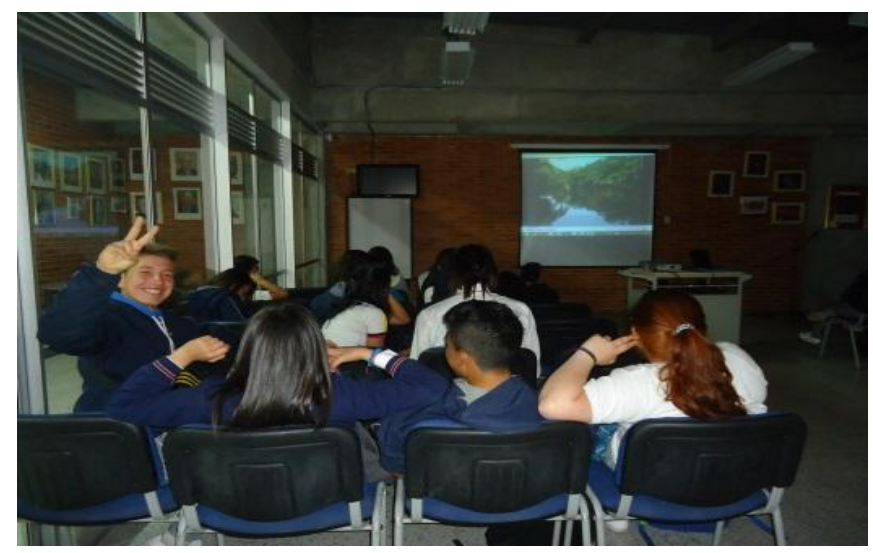

Imagen 1.Fotografía video lluvia ácida.

Finalizando se realizó la práctica de laboratorio donde se buscó presentar a los estudiantes simulaciones experimentales que nos permitan acercarnos a dichas problemáticas. Para esta investigación se realizó el siguiente procedimiento por parte de los estudiantes:

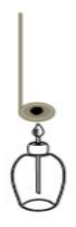

1)

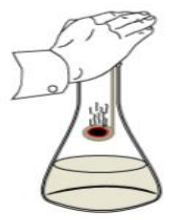

2)

Figura 1.Procedimiento para el desarrollo de la práctica.

Los principales resultados obtenidos de esta fase de investigación se categorizaron por los intereses, argumentación científica y principios de Educación Ambiental, los estudiantes comprenden las problemáticas ambientales y se interesen por la búsqueda de soluciones, durante el ejercicio los estudiantes se responsabilizaron de 
Edición Extraordinaria. p.p. 366 - 376

Memorias del VIII Encuentro Nacional de Experiencias en Enseñanza de la Biología y la Educación Ambiental. III Congreso Nacional de Investigación en Enseñanza de la Biología.

manera individual y colectiva para el manejo de su entorno inmediato, a través de posibles soluciones a diferentes problemáticas ambientales.

La experiencia lúdico científica permitió aprender disfrutando y motiva a los estudiantes de bachillerato a investigar y explorar las problemáticas ambientales. En este sentido, la propuesta investigativa contribuye a la apropiación de problemas globales, buscando desde sus conocimientos rutas para la formulación de posibles soluciones a las problemáticas ambientales, además durante la práctica los estudiantes fortalecieron los lazos humanos desde el trabajo en equipo. Algunos estudiantes en sus bitácoras de trabajo concluyeron que los ácidos de las precipitaciones tienen su origen en la combustión de los materiales fósiles necesarios para la obtención de energía (carbón, petróleo, gasolina). Dichas combustiones arrojan al ambiente grandes cantidades de gases, como dióxido de carbono, $\mathrm{CO}_{2}$, y óxidos de azufre, $\mathrm{SO}_{x}$, y de nitrógeno, $\mathrm{NO}_{x}$, que al combinarse con el vapor de agua que se encuentra en la atmósfera, se forman los ácidos que le confieren a la lluvia esa característica de precipitación ácida.

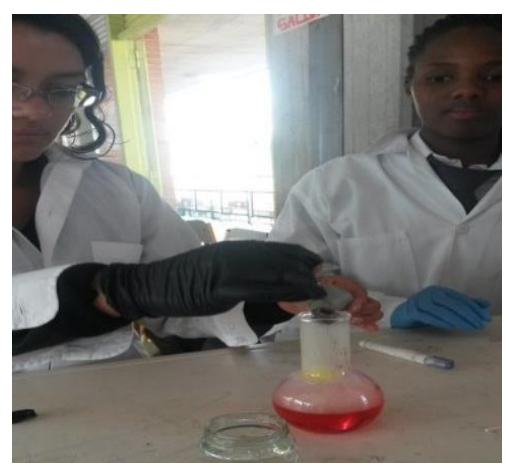

Imagen 2 .Fotografía práctica lluvia ácida

Los estudiantes expusieron sus ideas antes de la experimentación sobre algunas sustancias y la contaminación atmosférica por ejemplo, identifican que el dióxido de carbono es un gas de efecto invernadero, que a su vez este aumenta la temperatura 
Bio - grafia. Escritos sobre la Biología y su Enseñanza. ISSN 2027

Edición Extraordinaria. p.p. 366 - 376

Memorias del VIII Encuentro Nacional de Experiencias en Enseñanza de la Biología y la Educación Ambiental. III Congreso Nacional de Investigación en Enseñanza de la Biología.

global, produciendo el derretimiento de las capas polares y aumentando el nivel del mar, los estudiantes identifican las consecuencias del calentamiento global. Además, identificaron que el metano es un gas de efecto invernadero y realizan afirmaciones como el actual cambio climático es culpa del ser humano. Sin embargo no están en condiciones de explicar con sus propias palabras por lo menos los principios básicos del efecto invernadero o la lluvia ácida, tan solo los algunos estudiantes reconocen conceptos básicos, algunos conocen sus efectos negativos, los estudiantes saben que la industria, el transporte y la ganadería son fuentes contaminantes atmosféricas como el $\mathrm{SO}_{2}, \mathrm{NO}_{x}$ y $\mathrm{CH}_{4}$.

Asimismo, los estudiantes lograron identificar que la lluvia ácida puede producir daños en vegetación y edificios y que puede dañar aguas y suelos. A primera vista, la asignación de los gases responsables de la lluvia ácida no fue demasiado difícil entenderlo para los estudiantes, ya que los óxidos fueron un tema tratado con anterioridad $\mathrm{SO}_{2}$, el $\mathrm{CO}_{2}$ y los $\mathrm{NO}_{x}$. Por esto que la lluvia ácida haya sido entendida por una parte de los estudiantes sin embargo, las ecuaciones químicas de la lluvia ácida no fueron fáciles de formular.

La "contaminación atmosférica" es un concepto que lo asociaron con "el aire y las personas" la "lluvia ácida, con agujero de ozono, efecto invernadero y cambio climático" y con "peligro y enfermedad" como consecuencias. Los estudiantes no conocen bien el concepto suelen construir concepciones equivocadas, especular que el efecto invernadero no es natural, o en el caso de la lluvia ácida lo interpretan como un ácido que provoca enfermedades como cáncer o quemaduras en la piel y hasta deformidades, lo cual muestra desconocimiento en cuanto a los principios básicos de la lluvia ácida, y como una falta de diferenciación cognitiva de los temas de la lluvia ácida y la contaminación atmosférica.

\section{ACTIVIDADES RECONOCIMIENTO TERRITORIAL}

Luego de la experimentación se realizó la fase de investigación con actividades como RAE y la cartografía social, cada uno de los artículos adaptados corresponde a 
Memorias del VIII Encuentro Nacional de Experiencias en Enseñanza de la Biología y la Educación Ambiental. III Congreso Nacional de Investigación en Enseñanza de la Biología.

los temas de problemáticas ambientales y ciencias naturales. A continuación podemos establecer que cada uno tiene una etapa de desarrollo de preguntas y una etapa de comprensión del texto. Se adaptó un artículo titulado "Curtidores de San Benito se beneficiarían en más de seis mil millones de pesos" el cual fue tomado de la Secretaría Distrital de Ambiente. En este artículo las estudiantes pueden entender la relación que existe en entre los procesos químicos que suceden en las curtiembre en este caso las ubicadas en la cuenca alta del río Tunjuelo, localidad Tunjuelito, con la problemática ambiental de dicho sitio. A través de la nomenclatura inorgánica se logró reconocer los diferentes compuestos iónicos que perjudican la salud pública y el entorno así como las fuentes hídricas.

La aplicación de esta lectura en el tema de las curtiembres se trabajó a partir de las sesiones dirigidas, explorando una nueva alternativa entendiendo que el RAE no necesariamente tiene que ser escrito. Por lo tanto, se encontraron resultados significativos donde las estudiantes identifican la contaminación hídrica como un problema aledaño a ellas, donde sitúan cada uno de los compuestos químicos. Posteriormente se aplicó una cartografía social (CS) para reconocer la problemática en los contextos locales de los estudiantes, se buscó con la implementación del taller el reconocimiento del espacio físico y problemáticas ambientales en este caso el dibujo de su colegio con el recorrido de cada estudiante en donde podría observar la vegetación, la fauna y las actividades económicas relacionadas con la lluvia ácida.
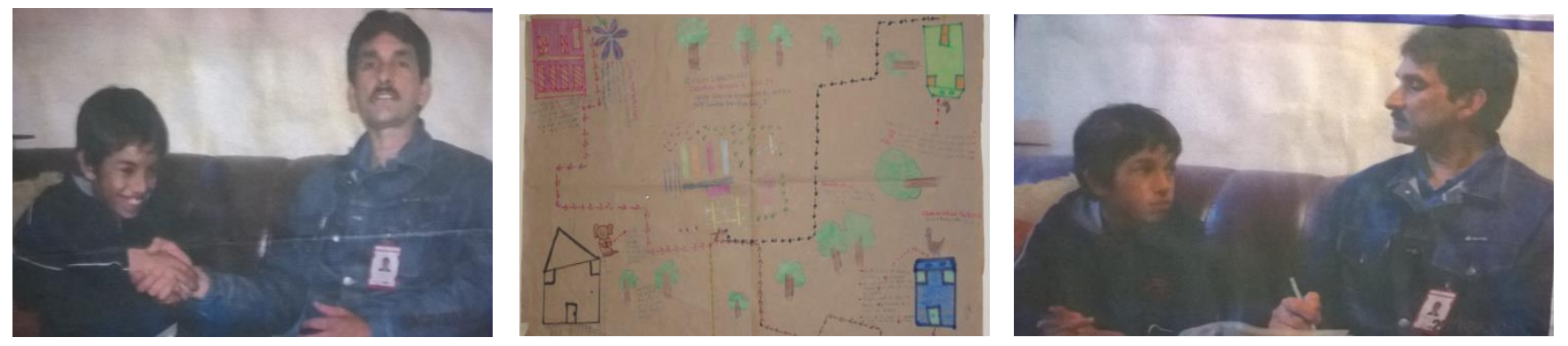

Figura 3 .Cartografía social, identificación de problemáticas ambientales locales 
Bio - grafia. Escritos sobre la Biología y su Enseñanza. ISSN 2027

Edición Extraordinaria. p.p. 366 - 376

Memorias del VIII Encuentro Nacional de Experiencias en Enseñanza de la Biología y la Educación Ambiental. III Congreso Nacional de Investigación en Enseñanza de la Biología.

Por tal motivo se buscó la aproximación de los estudiantes a las problemáticas que se encuentran cada vez más cerca de su colegio o viviendas y así identificarlas con su territorio buscando resultados con entrevistas a personas que trabajaran en industrias cercanas a la realidad de los estudiantes.

\section{CONCLUSIONES}

Los estudiantes se apropiaron de los temas mediante las diferentes actividades y práctica de laboratorio acerca de la lluvia ácida.

Se contribuyó al desarrollo de consciencia ecológica por parte de los estudiantes, generando discusiones reflexivas acerca del papel importante que juegan las prácticas humanas en la preservación del ambiente.

Permitir la participación en las discusiones sobre cuestiones de Ciencia y Tecnología es fundamental para identificar diferentes fortalezas y debilidades en las investigaciones realizadas en la enseñanza de la educación ambiental desde el enfoque de experimentación en el aula que se han llevado a cabo en diferentes contextos del país, donde se puede orientar el rumbo de futuras investigaciones.

\section{REFERENCIAS BIBLIOGRÁFICAS}

Centro de Ciencias de Sinaloa (2002). Cuaderno de experimentos de química para el salón de clases. Programa de seguimiento a los resolutivos de la sexta reunión de análisis. Culiacán, Sinaloa.

George E. Pataki. (2002). Lluvia Ácida La Amenaza Invisible. 25/07/2015, de departamento de conservación ambiental, Estado de Nueva York. Recuperado de sitio web: http://www.dec.ny.gov/docs/administration_pdf/spanishacidrain.pdf

You tuve. Video, lluvia acida recuperado de sitio web https://youtu.be/D80Idnh811I 
Bio - grafia. Escritos sobre la Biología y su Enseñanza. ISSN 2027

Edición Extraordinaria. p.p. 366 - 376

Memorias del VIII Encuentro Nacional de Experiencias en Enseñanza de la Biología y la Educación Ambiental. III Congreso Nacional de Investigación en Enseñanza de la Biología.

Granados, F. López, Á. Hernández, G. (2010). La lluvia ácida y los ecosistemas forestales. Revista Chapingo. Serie ciencias forestales y del ambiente versión impresa ISSN-0186-3231. Recuperado de sitio web http://www.scielo.org.mx/scielo.php?script=sci_arttext\&pid=S0186$\underline{32312010000200009}$

Diana M. González, Beatriz Zumalacárregui. (2003). juego de educación ambiental: laboratorio de productos químicos. Revista pedagogía universitaria, vol. 8.

Francisco Sóñora, Ma Mercedes Rodríguez-Ruibal y Raquel Troitiño. (2009). UN modelo activo de educación ambiental: prácticas sobre cambio climático. Enseñanza de las ciencias de la tierra. 